Article

\title{
Monitoring of Huntington's Disease Based on Wireless Sensing Technology
}

\author{
Qiyu Zhu ${ }^{1}$, Lei Guan ${ }^{2}$, Muhammad Bilal Khan ${ }^{1}$ and Xiaodong Yang ${ }^{1, *}$ \\ 1 School of Electronic Engineering, Xidian University, Xi'an 710071, China; qyzhu_xd@163.com (Q.Z.); \\ engrmbkhan1986@gmail.com (M.B.K.) \\ 2 School of Life Science and Technology, Xidian University, Xi'an 710071, China; 15926395470@163.com \\ * Correspondence: xdyang@xidian.edu.cn
}

Received: 17 December 2019; Accepted: 22 January 2020; Published: 27 January 2020

check for updates

\begin{abstract}
Huntington's disease (HD) is a rare genetic disorder that cannot be cured by current medical techniques. With the development of the disease, the life of patients will become more and more difficult. It is necessary to timely and effectively evaluate the development of the patient's condition based on the patient's clinical symptoms to help doctors to formulate a reasonable and effective treatment plan, alleviate the condition, and improve the quality of life, which reflects humane care. Currently, wearable devices or video surveillance are generally used to monitor the patients, and these schemes have some disadvantages. This paper presents a new method to monitor patients with HD using wireless sensing technology. Firstly, experimental data were collected by the self-developed microwave sensing platform (MSP), and then the data were preprocessed. Finally, support vector machine (SVM) and random forest (RF) algorithms were used to train the model. The MSP system continuously monitors patients in a non-contact way, which will not bring inconvenience to patients' lives, and will not involve privacy issues. The experimental results show that the prediction accuracy of SVM can be as high as $98.0 \%$ and that of RF can be as high as $96.7 \%$, which proves the feasibility of the technical scheme described in this paper.
\end{abstract}

Keywords: HD; MSP; RF; SVM; wireless sensing technology

\section{Introduction}

Huntington's disease (HD) is a very rare autosomal dominant genetic disease. The cause of the disease is the mutation of Huntington gene on chromosome 4 of the patient, resulting in the variation of protein, which leads to the change of normal neural function through the related molecular mechanism [1]. The diagnosis of this disease depends on genetic testing. It usually develops around 35-45 years old [2]. The disease degenerates the patient's physical and psychological intelligence during the working-age and places a heavy burden on the patient. The clinical symptoms of HD mainly fall into three categories: motor symptoms, cognitive symptoms, and mental symptoms [3-5]. The typical manifestation of motor symptoms is that the fingers appear to play piano-like movements, accompanied by weird facial expressions. If the trunk is involved, the patient can have a dance-like gait. As the disease progresses, other body movements will also become slow and uncoordinated. In the end, the patient's entire system will be affected, making it difficult for the patient to complete simple daily actions such as walking, talking, eating, dressing, and washing. Cognitive symptoms sometimes appear many years earlier than motor symptoms. In the early stages of cognitive symptoms, patients do not only suffer from episodic memory, but also have significant functional dysfunction and do not understand the meaning of the speaker's language. As the disease progresses, the patient's dementia symptoms worsen. However, even if the condition is severe, the patient retains some cognitive functions. The third type of symptoms are mental symptoms. The mental symptoms of 
patients are often earlier than or synchronous with abnormal motor symptoms, mainly manifested as depression, often accompanied by insomnia, anorexia, and personality changes. In the later stage, patients will gradually experience hallucinations, delusions, paranoia, and aggressive behavior.

HD is characterized by complex clinical symptoms, progressive deterioration of the patient's condition, and usually death 15-20 years after onset [6]. At present, there is no effective treatment for this disease. The purpose of treatment is to improve the quality of life of patients. Several existing treatment methods can be classified as cause treatment and symptomatic treatment [7]. Cause treatment includes direct gene therapy and other indirect molecular therapy, this method cannot be realized at present, but a lot of research has been carried out. The symptomatic treatment is that the doctor obtains the clinical symptoms of the patient through visual observation and other methods and diagnoses the patient's condition against the HD Scale [8], so as to formulate a drug treatment plan to directly alleviate the patient's symptoms.

Drug therapy is currently the main treatment for HD. In order to confirm whether drug therapy is effective, doctors need to continue to follow up the effect of drug therapy, so as to improve the treatment. But this treatment has a disadvantage; the doctor's judgment of the patient is mainly based on the naked eye and the HD Scale. The diagnosis result can easily be affected by the subjective consciousness of the doctor. A promising solution is needed to continuously monitor the patient and provide an objective diagnosis basis. At the same time, the mental symptoms of patients are unstable, and patients are prone to hallucinations and aggressive behavior. In order to avoid self-injury, we also need to carry out continuous monitoring and give timely psychotherapy to patients.

In order to solve the above-mentioned problems, this paper proposes a new method to monitor HD patients using wireless sensing technology and demonstrates the feasibility of the proposed method. We independently developed the microwave sensing platform (MSP) monitoring tool, which is composed of a transmitter module and a receiver module. It can be directly installed in the indoor environment, without any contact with the patient, and it can be completely monitored in a non-contact way. The working process of MSP is: the transmitting module transmits the wireless signal in C-band, the receiving module receives the wireless signal and extracts the channel state information (CSI) data through channel estimation. After collecting CSI data, we first carried out a series of data preprocessing steps, including removing outliers and wavelet transform de-noising. Then we extracted the features of the preprocessed data to make the sample set. Finally, we used support vector machine (SVM) [9] and random forest (RF) [10] to train the classification model, respectively. The trained classification model can effectively distinguish the behavior of HD patients and normal people. The experimental results show that the accuracy of SVM and RF is $98.0 \%$ and $96.7 \%$, respectively, which proves that the methods described in this paper can effectively monitor HD patients.

The rest of this paper is organized as follows. The Section 2 will introduce the current research on the monitoring of $\mathrm{HD}$ and make a brief comparison with the experimental scheme proposed in this paper. In the Section 3, we will introduce the principle of wireless sensing technology, and in the Section 4, we will describe the experimental scheme design. In the Section 5, we will describe the data processing flow. In the Section 6, we will discuss the experimental results and draw conclusions in the Section 7.

\section{Related Work}

At present, a large number of scholars have carried out related research on monitoring patients with HD using wearable devices. For example, Dinesh et al. reported a preliminary study to analyze motor symptoms associated with HD and Parkinson's disease based on sensor signal detection and data analysis. They use a light-weight, low-power sensor to monitor the motor symptoms of patients. The sensor adheres to the limbs and chest of patients like a tattoo and can continuously monitor patients for 48 hours. The experimental results show that the sensor can capture different clear signals of different clinical symptoms [11]. Bennasar et al. proposed an HD upper limb dyskinesia evaluation system. A triaxial acceleration sensor was worn on each wrist and chest of the experimental participants. 
The collected sensor data was used to develop an automatic classification system to distinguish normal people from HD patients [12,13] proposed a method of using inertial sensors to identify healthy gait, HD patient gait, and hemiplegic patient gait. This method is based on a supervised and trained two-state hidden Markov model, which can be extended to different research subjects for clinical practice and personal health assessment. At the same time, Francisco et al. collected the gait data of HD patients by binding the iPhone on the ankles of patients, using the built-in smart sensor of iPhone, and classified the data with the general assembly (meta) classifier algorithm to distinguish normal people and HD patients [14]. The use of wearable devices to monitor the movements and gaits of HD patients requires the binding of electronic devices to a part of the patient's body, which will affect the patient's movements to a certain extent and cannot collect movement data in the natural state of the patient. At the same time, it will also affect the patient's living comfort. Some scholars have also proposed the idea of using a camera to monitor patient activity, combined with related video image processing algorithms to extract patient activity information. For example, Agrawal et al. proposed a method for human fall detection based on video surveillance, but this may violate the privacy of patients [15].

As far as the authors know, this paper proposes for the first time to use C-band wireless sensing technology to monitor HD patients completely in a non-contact way for continuous monitoring. Compared with the traditional monitoring scheme, it has the following characteristics:

(1) Monitoring patients completely in a non-contact way will not bring discomfort to the patient's body, and data can be collected under natural conditions.

(2) Continuous monitoring of the patient's condition by wireless sensing will not affect the patient's privacy.

(3) MSP has no requirements for the working environment and is easy to install.

(4) The collected data are processed, and two machine learning algorithms are used to train the model. The two machine learning algorithms can be compared with each other, which makes the experimental results more convincing.

\section{Principle of Wireless Sensing Technology}

The proposed technical scheme is suitable for non-contact continuous monitoring of HD patients in an indoor environment. Therefore, this section will first describe the indoor model of wireless signal propagation in detail and explain the principles behind wireless sensing technology. Since this article mainly collects CSI data through MSP, we will next reveal the nature of CSI data, derive its mathematical expression, and describe MSP in detail at the end of this section.

\subsection{Indoor Propagation Model of Wireless Signal}

When the wireless signal propagates in the indoor environment, it is influenced by the obstacles in the propagation path, which cause reflection and diffraction. After the propagation of different paths, each component reaches the receiving end with different strengths and phases, resulting in a multipath effect [16]. The indoor propagation model of the wireless signal is shown in Figure 1. 


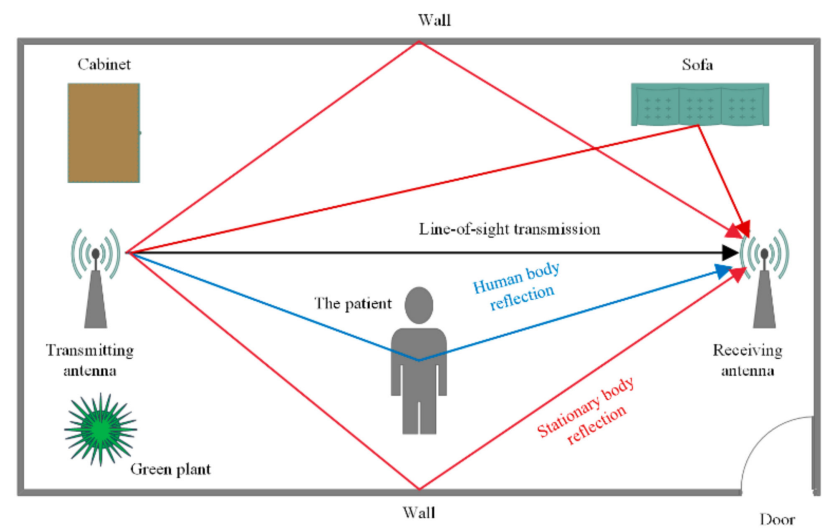

Figure 1. Indoor propagation model of wireless signal.

According to the Fries transfer formula [17], the receiving power of the receiving antenna can be expressed as:

$$
P_{r}=\frac{P_{t} G_{t} G_{r} \lambda^{2}}{(4 \pi R)^{2}}
$$

where $P_{t}$ and $P_{r}$ are the power of transmitting antenna and receiving antenna, respectively; $G_{t}$ and $G_{r}$ are the gain of transmitting antenna and receiving antenna, respectively; and the distance between transmitting antenna and receiving antenna is $R . \lambda$ is the wavelength of electromagnetic wave.

Assuming that the propagation path length of the electromagnetic wave through the interference of static objects is $D$, and the propagation path length through the interference of the human body is $X$, the Fries transfer formula can be rewritten as follows.

$$
P_{r}=\frac{P_{t} G_{t} G_{r} \lambda^{2}}{16 \pi^{2}\left(R^{2}+D^{2}+X^{2}\right)}
$$

In Formula (2), we can see that both $R$ and $D$ do not change. When people are indoors, $X$ will change, resulting in a change in the power of the receiving antenna. At the same time, because the signal phase is a linear function of the distance of the propagation path, the change of the propagation path will also lead to the change of the signal phase [18]. Human behavior changes the strength and phase of the signal, and the CSI describes the loss and fading on the transmission path. When there is a moving target between the transmitting and receiving devices, the wireless signal reflected by the moving target increases the dynamic component of the channel. The fluctuation of the channel corresponds to the motion information of the target one by one. We can get CSI data from the signals collected by the receiving devices. By analyzing the CSI data, we can sense the changes of the external environment. In the next section, we explain the essence of CSI and its mathematical expression [19].

\subsection{CSI}

In order to eliminate the adverse effect of a multipath effect on wireless signal transmission, the MSP described in this paper uses orthogonal frequency division multiplexing (OFDM) modulation technology to decompose the data stream to be transmitted into several independent sub-data streams, that is, multiple subcarriers, and then transmits them in parallel, which can effectively eliminate the inter-symbol interference caused by the multipath effect in high-speed data stream transmission. At the same time, OFDM modulation technology can also greatly improve the data transmission efficiency. Because there are multiple subcarriers, each subcarrier channel is independently available, which also increases the amount of data to extract more information [20]. 
Multiple input multiple output (MIMO) is supported by OFDM modulation technology [21]. The channel model of the MIMO system in the frequency domain can be expressed as

$$
Y=H X+N
$$

where $Y$ represents the receiving signal, $X$ represents the transmitting signal, $N$ represents the environmental noise, and $\boldsymbol{H}$ represents the state matrix of the wireless channel, and its dimensions are $N_{T} \times N_{R} \times N_{C} . N_{T}, N_{R}$, and $N_{C}$, respectively, represent the number of transmitting antennas, receiving antennas, and subcarriers.

CSI is essentially a representation of the frequency response of each subcarrier channel. For each independent subcarrier channel, its frequency response can be expressed as

$$
H_{k}\left(f_{k}\right)=\left\|H_{k}\left(f_{k}\right)\right\| e^{j a r g}\left(H_{k}\left(f_{k}\right)\right)\left(1 \leq k \leq N_{C}\right)
$$

where $f_{k}$ represents the center frequency of the Kth subcarrier, $\left\|H_{k}\left(f_{k}\right)\right\|$ represents the CSI amplitude information of the Kth subcarrier, and $\arg \left(H_{k}\left(f_{k}\right)\right)$ represents the CSI phase information of the Kth subcarrier.

After continuous data collection over a period of time, CSI data can be obtained through the channel estimation formula [22].

$$
\hat{H} \approx \frac{Y}{X}
$$

\subsection{MSP}

The MSP independently developed in this paper works in C-band $(4.8 \mathrm{GHz})$. It is a highly customizable platform that can adapt to different application scenarios. MSP consists of omnidirectional antenna, industrial personal computer, absorbing material, frequency converter, and other related facilities. The use of absorbing materials is mainly to shield the surrounding environment from interference. The main function of MSP is to obtain the CSI of the wireless channel. By analyzing the CSI data, the behavior of patients can be monitored.

MSP uses OFDM technology. Its essence is an OFDM transceiver system, and its functional block diagram for obtaining CSI is shown in Figure 2.

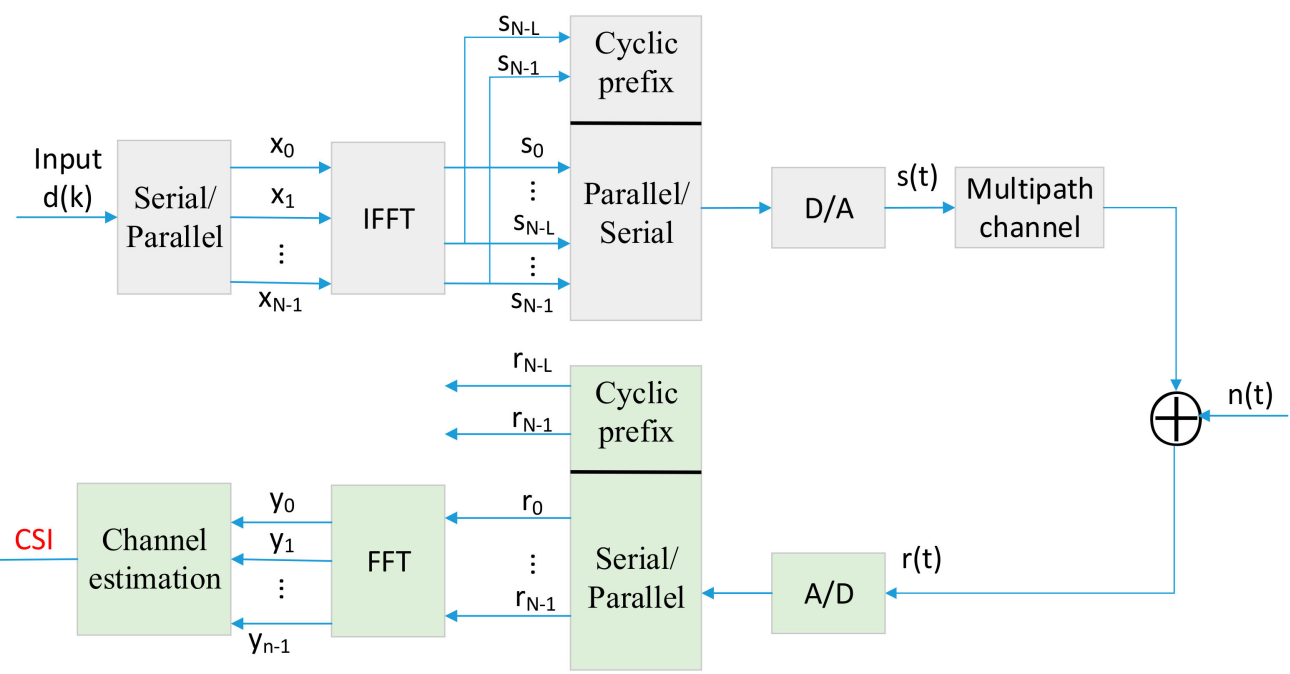

Figure 2. Block diagram of microwave sensing platform (MSP) to obtain channel state information (CSI) data.

In Figure 2, $d(k)$ is converted to $\mathrm{N}$ parallel data $\left\{x_{0}, x_{1}, \ldots, x_{N-1}\right\}$ through serial-parallel conversion. These data can be regarded as $\mathrm{N}$ data in the frequency domain. A set of time domain data 
$\left\{s_{0}, s_{1}, \ldots, s_{N-1}\right\}$ obtained after the inverse discrete Fourier transform (IDFT) is an OFDM symbol. After adding a cyclic prefix to an OFDM symbol, the OFDM symbols are transmitted on the wireless multipath channel after parallel-serial conversion and digital-analog conversion. At the receiving end, the reverse work is performed: analog-digital conversion, parallel-serial conversion, removing cyclic prefix, and fast Fourier transform (FFT). The training sequence after FFT is used to perform channel estimation according to Equation (5), and CSI data can be obtained.

\section{The Experimental Scheme}

The purpose of this article is to continuously monitor patients with HD. To distinguish the patient's actions from other normal daily movements, so as to provide an objective clinical diagnosis basis for doctors and facilitate doctors to make appropriate treatment plans. The experimental flow chart is shown in Figure 3.

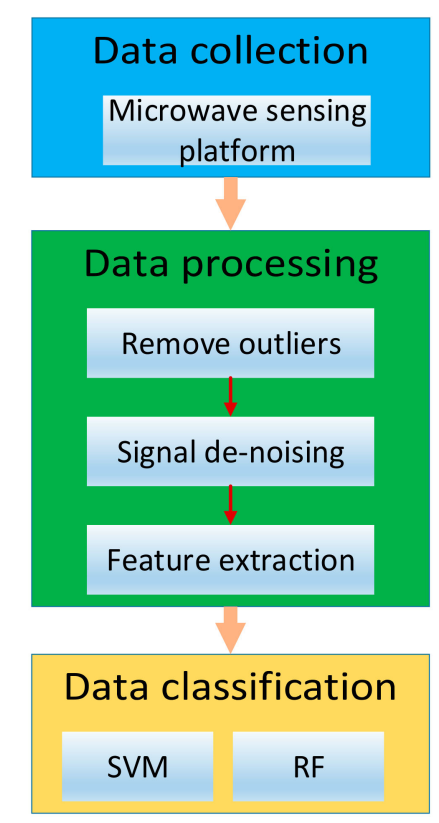

Figure 3. The experiment flow chart.

This experiment will collect the data of several actions like normal standing, normal sitting, normal walking, standing of HD, sitting of HD, and gait of HD, as shown in Table 1.

Table 1. The actions used in the experiment.

\begin{tabular}{cc}
\hline No. & Action \\
\hline 1 & Normal \\
2 & standing \\
3 & Normal sitting \\
4 & Standing of HD \\
5 & Sitting of HD \\
6 & Gait of HD \\
\hline
\end{tabular}

The simulation experiment was carried out in a laboratory in the new science and technology building of Xidian University, which is $7 \mathrm{~m} \times 5 \mathrm{~m}$ in size. The transmitting and receiving antennas of the MSP were respectively placed at two ends of the laboratory, with a horizontal distance of $4 \mathrm{~m}$. The transmitting antenna and receiving antenna were positioned $1.8 \mathrm{~m}$ from the ground and $1.2 \mathrm{~m}$ from the ceiling. The transmitting module of the MSP was composed of a control computer with a 
wireless adapter and an omnidirectional antenna. The wireless adapter was configured in an injection mode for sending wireless signals. The receiving module of the MSP consisted of a control computer with a wireless adapter and three omnidirectional antennas. The wireless adapter was configured in a listening mode for receiving signals and extracting CSI data. The MSP operated in C-band $(4.8 \mathrm{GHz})$ and used OFDM technology to modulate the signal with a total of 30 subcarriers. The signal bandwidth was set at $20 \mathrm{MHz}$. The transmitting antenna had a packet frequency of $200 \mathrm{~Hz}$, and the time window was $12 \mathrm{~s}$. We collected 300 samples for each action. The experimental scene is shown in Figure 4.

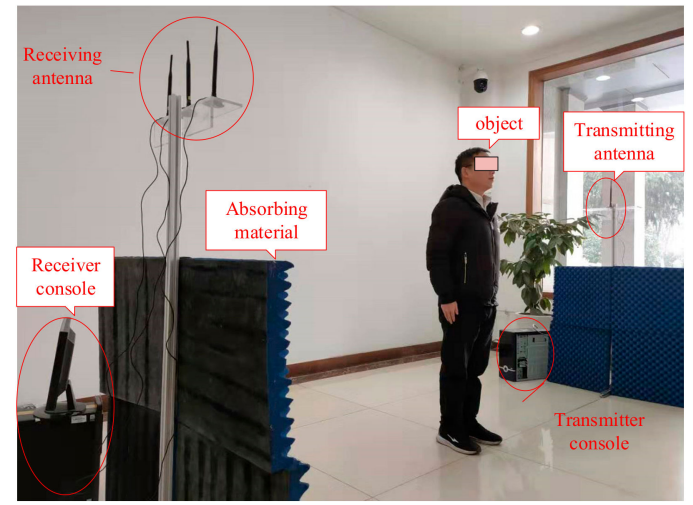

(a)

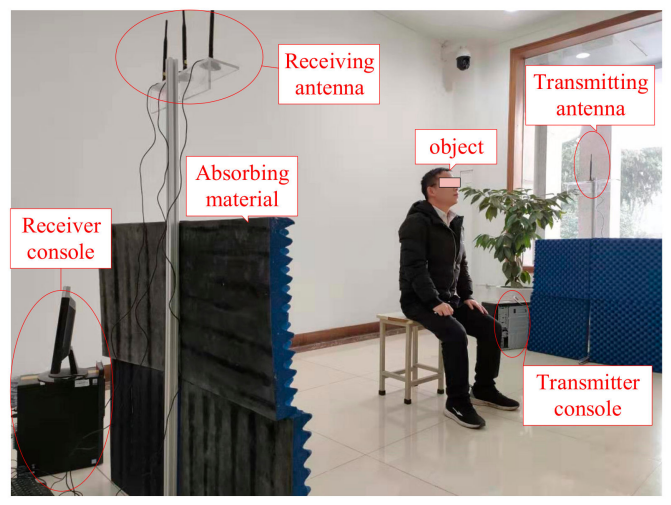

(b)

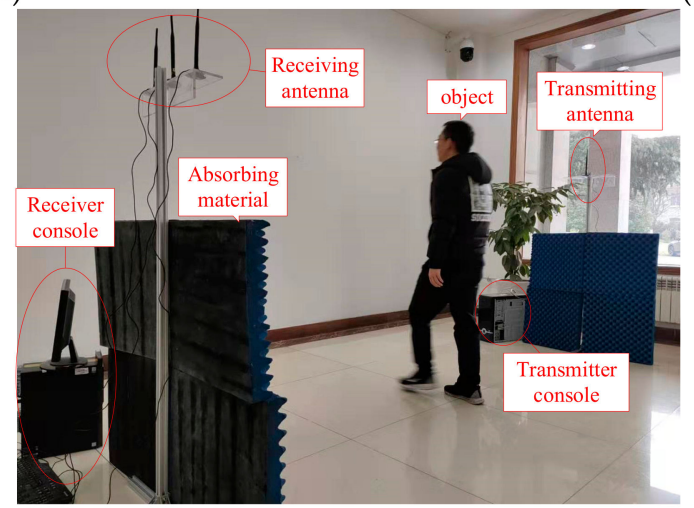

(c)

Figure 4. The experimental scene: (a) standing; (b) sitting; (c) walking.

In Figure 4c, the object moves in a direction perpendicular to the line connecting the transmitter and receiver, mainly considering that if the object moves along the line from the transmitter to the receiver, the line-of-sight transmission will be weakened, and most of the energy will be lost, resulting in a reduction of signal amplitude at the receiver, which is not conducive to any kind of communication. At the same time, in clinical trials, the movement tasks of patients are usually specified, so we chose a movement mode that is more conducive to study the feasibility of the technical scheme described in this article.

In this work, our research focuses on the feasibility of using wireless sensing technology to monitor HD patients, and the number of HD patients is very small [23]; hence, we did not recruit real patients, but volunteers from our team simulated HD movements.

Before the experiment, we fully informed the experimental participants of all relevant matters and contents of the clinical experiment. By watching videos of clinical manifestations of HD patients and reading related literature, all experimental participants were rigorously trained to simulate real HD patient movements.

Due to the limited staff in our team, there were a total of 10 volunteers who participated in our experiment, including 6 males and 4 females, aged between 24 and 48 years. Details of volunteers are shown in Table 2. 
Table 2. Details of volunteers.

\begin{tabular}{ccccc}
\hline ID & Age & Gender & Weight $\mathbf{( k g )}$ & Height $(\mathbf{c m})$ \\
\hline 1 & 26 & Male & 72 & 181 \\
2 & 45 & Male & 53 & 173 \\
3 & 30 & Male & 64 & 168 \\
4 & 32 & Female & 50 & 155 \\
5 & 24 & Female & 56 & 165 \\
6 & 25 & Male & 62 & 164 \\
7 & 36 & Male & 70 & 174 \\
8 & 48 & Male & 65 & 175 \\
9 & 25 & Female & 51 & 161 \\
10 & 40 & Female & 57 & 163 \\
\hline
\end{tabular}

We randomly selected 5 volunteers ( 3 male and 2 female) to simulate the movements of HD patients, and the remaining 5 volunteers ( 3 male and 2 female) were used as normal reference. Each set of actions was repeated 60 times for each volunteer, and it took 6 days to collect the data.

\section{The Data Processing}

After collecting CSI data, we needed to perform a series of processing steps on the data. In this section, we follow the steps shown in Figure 3 to process the collected CSI data in turn.

\subsection{Data Preprocessing}

\subsubsection{Remove Outliers}

In the process of data collection, due to the influence of environmental noise or internal voltage fluctuation of the device, there was a large number of outliers in the original signal. These outliers seriously distort the original signal and must be removed.

We used the "Hampel" function in MATLAB to remove the outliers of the original signal. For each sampling point of the original signal, the function calculates the median of the window consisting of the sampling point and the three sampling points on the left and right sides. Then the absolute value of the median is used to estimate the standard deviation of the median at each sampling point. If a sample is more than three standard deviations away from the median, the sample is replaced with the median [24]. The outliers contained in the original signal are shown in Figure 5. The original signal after removing the outliers is shown in Figure 6.

Outliers can affect signal de-noising. After removing the outliers of the original signal, we can de-noise the signal, which is described in the next section.

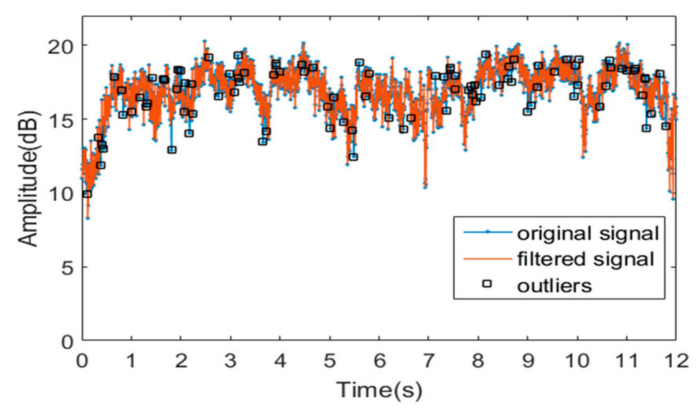

Figure 5. Outliers contained in the original signal. 


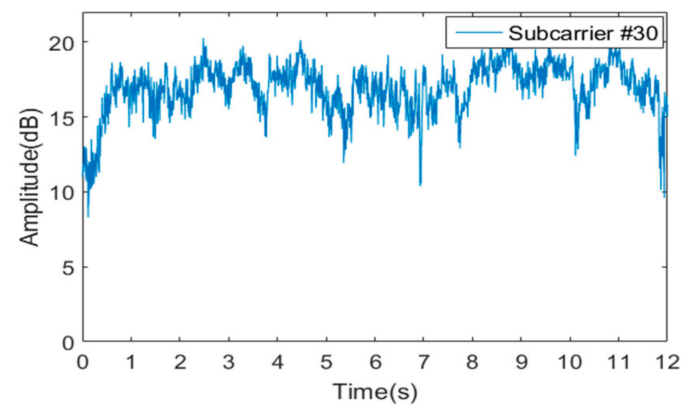

Figure 6. The original signal after removing the outliers.

\subsubsection{Signal De-Noising}

The patient's actions mainly affect the low-frequency components of the wireless signal, so we needed to filter out the high-frequency noise generated by environmental noise, slight internal voltage fluctuations, etc. In this paper, wavelet transform was used to realize signal de-noising [25]. The "wden" function with one-dimensional noise reduction in the MATLAB toolbox was used. The main principle of this function is to filter out noise through threshold processing of the wavelet decomposition coefficient of the original signal.

We used the "sym8" wavelet to decompose the original signal in 5 layers. The "SimN" $(\mathrm{N}=2,3$, $\ldots, 8)$ wavelet has good symmetry, which can reduce the phase distortion during signal decomposition and reconstruction to a certain extent. At the same time, we applied heuristics to overcome the problem of noise distribution at each decomposition level. The signal waveforms of each action after de-noising by wavelet transform are shown in Figure 7; the larger the variance, the larger the information. We chose the subcarrier according to the principle of maximum variance [26].

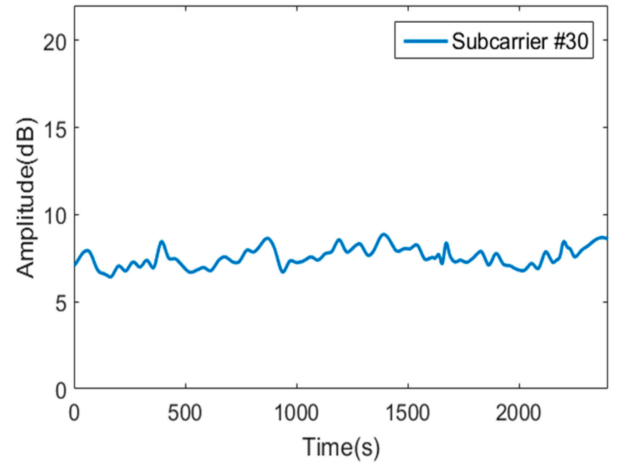

(a)

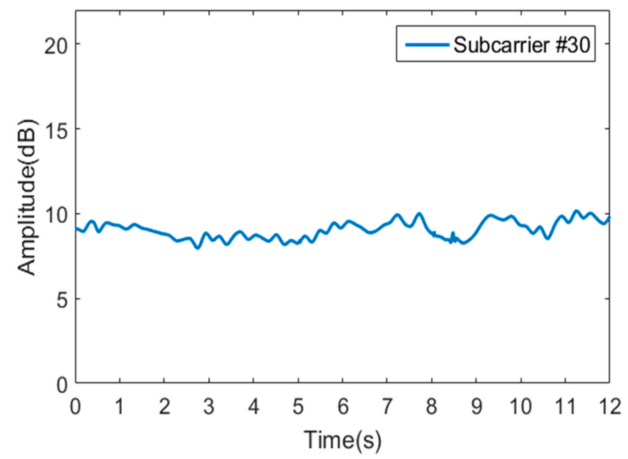

(c)

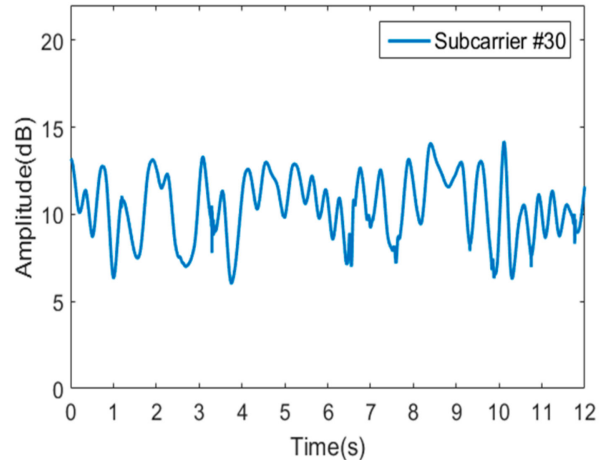

(b)

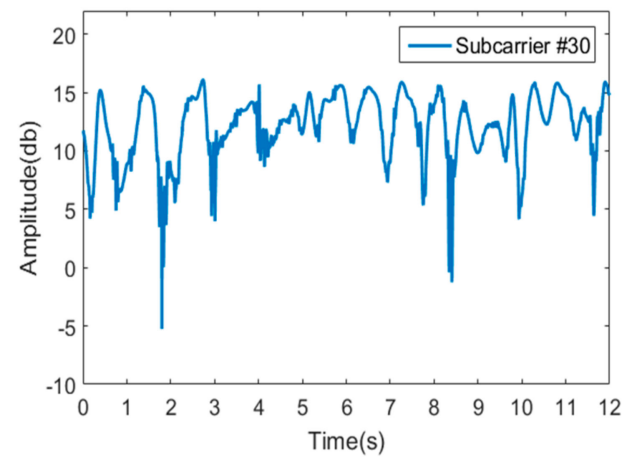

(d)

Figure 7. Cont. 


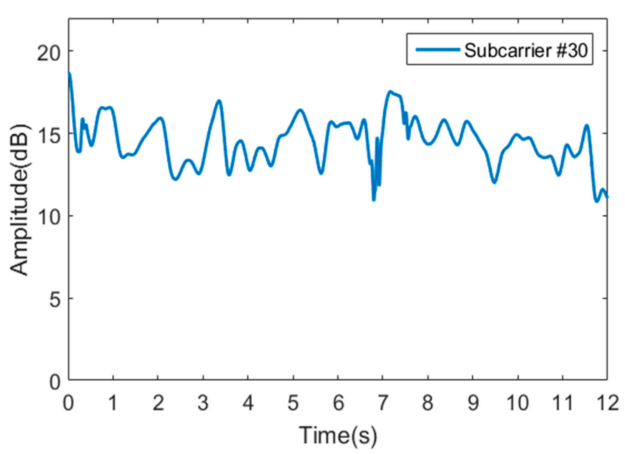

(e)

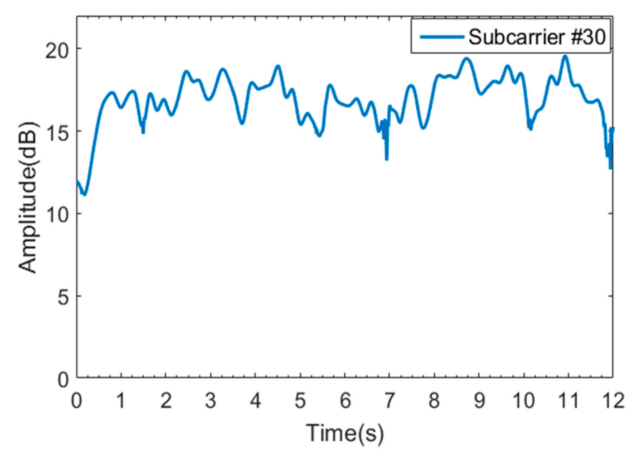

(f)

Figure 7. The waveforms of each action after data preprocessing: (a) normal standing; (b) standing of Huntington's disease (HD); (c) normal sitting; (d) sitting of HD; (e) normal walking; (f) gait of HD.

\subsection{Feature Extraction}

It can be seen from Figure 7 that the time domain waveform of each action is quite different. We extracted eight time domain features from the signal waveform of each action, as shown in Table 3.

Table 3. Time domain features.

\begin{tabular}{cc}
\hline Features & A Formula to Calculate \\
\hline Mean value & $Y_{M V}=\frac{1}{N} \sum_{i=1}^{N} x_{i}$ \\
Standard deviation & $Y_{S D}=\sqrt[2]{\frac{1}{N-1} \sum_{i=1}^{N}\left(x_{i}-Y_{M V}\right)^{2}}$ \\
Root mean square & $Y_{R M S}=\sqrt[2]{\frac{1}{N} \sum_{i=1}^{N} x_{i}^{2}}$ \\
Peak-to-peak value & $Y_{P P V}=\max \left(x_{i}\right)-\min \left(x_{i}\right)(i=1,2, \ldots, N)$ \\
Kurtosis & $Y_{K}=\frac{\frac{1}{N} \sum_{i=1}^{N}\left(\left|x_{i}\right|-Y_{M V}\right)^{4}}{Y_{R M S}{ }^{4}}$ \\
Skewness & $Y_{S}=\frac{\frac{1}{N} \sum_{i=1}^{N}\left(\left|x_{i}\right|-Y_{M V}\right)^{3}}{Y_{R M S}{ }^{3}}$ \\
Peak factor & $Y_{P}=\frac{\max \left(x_{i}\right)}{Y_{R M S}}(i=1,2, \ldots, N)$ \\
Waveform factor & $Y_{W}=\frac{N * Y_{R M S}}{\sum_{i=1}^{N}\left|x_{i}\right|}(i=1,2, \ldots, N)$ \\
\hline
\end{tabular}

\subsection{Model Training}

We used SVM and RF to train the model to ensure the accuracy of data classification and to determine which algorithm has a better effect in practical applications. At the same time, in order to make the training model reliable, we used the four-fold cross validation method to divide the data set.

We selected the radial basis function (RBF) as the kernel function of SVM, and the RF contained 500 decision trees.

\section{Result and Discussion}

The confusion matrix of each classification algorithm is shown in Table 4 . 
Table 4. Confusion matrix of classification algorithms.

\begin{tabular}{|c|c|c|c|c|c|c|c|}
\hline \multirow{2}{*}{$\begin{array}{l}\text { Classification } \\
\text { Algorithm }\end{array}$} & \multirow{2}{*}{ Actual Action } & \multicolumn{6}{|c|}{ Predict Action (Number of Samples) } \\
\hline & & Standing & Sitting & Walking & Standing of HD & Sitting of HD & Gait of HD \\
\hline \multirow{6}{*}{ SVM } & Standing & 73 & 0 & 2 & 0 & 0 & 0 \\
\hline & Sitting & 1 & 74 & 0 & 0 & 0 & 0 \\
\hline & Walking & 2 & 0 & 72 & 0 & 0 & 1 \\
\hline & Standing of HD & 0 & 0 & 0 & 75 & 0 & 0 \\
\hline & Sitting of HD & 0 & 0 & 0 & 0 & 75 & 0 \\
\hline & Gait of HD & 0 & 0 & 1 & 0 & 2 & 72 \\
\hline \multirow{6}{*}{ RF } & Standing & 72 & 1 & 0 & 2 & 0 & 0 \\
\hline & Sitting & 0 & 75 & 0 & 0 & 0 & 0 \\
\hline & Walking & 0 & 0 & 75 & 0 & 0 & 0 \\
\hline & Standing of HD & 4 & 0 & 0 & 71 & 0 & 0 \\
\hline & Sitting of HD & 0 & 0 & 0 & 1 & 70 & 4 \\
\hline & Gait of HD & 0 & 0 & 2 & 0 & 1 & 72 \\
\hline
\end{tabular}

The experimental accuracy of each algorithm is shown in Figure 8.

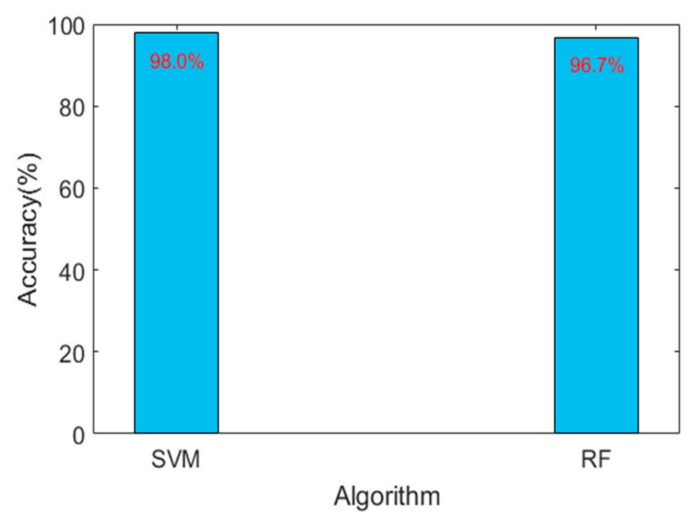

Figure 8. Algorithm accuracy.

Figure 7a-d shows different waveforms of normal people and patients with HD under static action. It can be seen from the figure that the static action signal waveform of normal people is relatively gentle, while the static action signal waveform of patients fluctuates greatly because patients with HD have convulsions when they are ill and dance-like movements when they are serious. Figure 7e,f shows the signal waveforms of normal gait and gait in patients with HD. Due to the large amplitude of walking itself, the abnormal body swing of patients with HD may be covered by the walking movement, resulting in the signal waveform discrimination between normal gait and abnormal gait not being very obvious, which is consistent with reality. At the same time, we can see from Figure 7 that the differences between the action signal waveform of normal people and patients with HD are obvious in the time domain. In order to reduce the amount of data and improve the efficiency of the algorithm training model, we extracted eight time domain features from the samples, which can well describe the time domain waveform, and the experimental results also prove this.

Table 4 is the confusion matrix of SVM and RF. It can be seen that the SVM algorithm can completely distinguish the static actions of patients with HD, while the normal static actions have misclassification, which shows that the performance of patients in the static actions is inconsistent, the performance of body convulsion or dance is different, and the performance of normal people in static actions are consistent. It can also be seen that RF can completely distinguish between normal gait and normal sitting action. Both SVM and RF cannot distinguish the normal gait and the abnormal gait of patients with HD completely, because the time domain signal waveform of them is similar.

Figure 8 shows the prediction accuracy of the two algorithms. The prediction accuracy of SVM is $98.0 \%$, and that of RF is $96.7 \%$. Both algorithms can achieve high prediction accuracy, which proves that the experimental scheme described in this paper is feasible. At the same time, we can draw a 
conclusion that when using the method described in this paper to distinguish the actions of patients with HD, the performance of SVM is better than that of RF.

\section{Conclusions}

As far as we know, this article first proposed a method for monitoring HD patients using wireless sensing technology and studied the feasibility of the proposed technical scheme in depth. We used the self-developed MSP to collect CSI data, then removed the outliers, and filtered the CSI data with the wavelet transform. After that, we extracted eight time domain features from each action data set and trained the model with SVM and RF machine learning algorithms. The experimental results show that the prediction accuracy of the SVM algorithm can reach $98.0 \%$, and the prediction accuracy of the RF algorithm can reach $96.7 \%$. Both algorithms can effectively distinguish the normal actions and the actions of patients with HD, which proves that the technical scheme described in this paper is feasible. This will provide a basis for doctors to objectively diagnose patients' conditions. At the same time, the technology can help doctors to follow up on the patient's condition development and improve the treatment plan in time. At present, the MSP described in this paper is not fully automated but also needs some manual operations. Next, we will continue to improve the experimental platform to make it fully automated to achieve data collection, data processing, and data analysis in a one-click operation. At the same time, we will also further explore the application of wireless sensing technology in medical care.

Author Contributions: Conceptualization, Q.Z.; methodology, L.G.; resources, X.Y.; writing-original draft preparation, Q.Z.; writing—review and editing, M.B.K.; supervision, X.Y.; project administration, X.Y.; funding acquisition, X.Y. All authors have read and agreed to the published version of the manuscript.

Funding: This research was funded by the National Natural Science Foundation of China grant number 61301175.

Conflicts of Interest: The authors declare no conflict of interest.

\section{References}

1. Oliveira, J.M.A. Nature and cause of mitochondrial dysfunction in Huntington's disease: focusing on huntingtin and the striatum. J. Neurochem. 2010, 114, 1-12. [CrossRef] [PubMed]

2. Walker, F.O. Huntington's disease. Semin. Neurol. 2007, 27, 143-150. [CrossRef] [PubMed]

3. Shannon, K.M. Huntington's disease-Clinical signs, symptoms, presymptomatic diagnosis, and diagnosis. Handb. Clin. Neurol. 2011, 100, 3-13. [PubMed]

4. Goodman, L.V.; Cha, J.; Como, P. Poster 24: Expert Treatment Preferences for the Motor, Mood, and Behavioral Symptoms of Huntington's Disease. Neurotherapeutics 2009, 6, 211-212.

5. Hofmann, N. Understanding the Neuropsychiatric Symptoms of Huntington's Disease. J. Neurosci. Nurs. 1999, 31, 309-313. [CrossRef] [PubMed]

6. Brackenridge, C.J. The relation of type of initial symptoms and line of transmission to ages at onset and death in Huntington's disease. Clin. Genet. 1971, 2, 287-297. [CrossRef] [PubMed]

7. Bonelli, R.M.; Hofmann, P. A systematic review of the treatment studies in Huntington's disease since 1990. Expert Opin. Pharmacother. 2007, 8, 141-153. [CrossRef] [PubMed]

8. Huntington Study Group. Unified Huntington's disease rating scale: Reliability and consistency. Mov. Disord. 1996, 11, 136-142. [CrossRef] [PubMed]

9. Widodo, A.; Yang, B.-S. Support vector machine in machine condition monitoring and fault diagnosis. Mech. Syst. Signal Process. 2008, 21, 2560-2574. [CrossRef]

10. Breiman, L. Random Forests. Mach. Learn. 2001, 45, 5-32. [CrossRef]

11. Dinesh, K.; Xiong, M.; Adams, J.; Dorsey, R.; Sharma, G. Signal analysis for detecting motor symptoms in Parkinson's and Huntington's disease using multiple body-affixed sensors: A pilot study. In Proceedings of the 2016 IEEE Western New York Image and Signal Processing Workshop (WNYISPW), Rochester, NY, USA, 18 November 2016; pp. 1-5. 
12. Bennasar, M.; Hicks, Y.A.; Clinch, S.P.; Jones, P.; Holt, C.; Rosser, A.; Busse, M. Automated Assessment of Movement Impairment in Huntington's Disease. IEEE Trans. Neural Syst. Rehabil. Eng. 2018, 26, 2062-2069. [CrossRef] [PubMed]

13. Mannini, A.; Trojaniello, D.; della Croce, U.; Sabatini, A.M. Hidden Markov model-based strategy for gait segmentation using inertial sensors: Application to elderly, hemiparetic patients and Huntington's disease patients. In Proceedings of the 2015 37th Annual International Conference of the IEEE Engineering in Medicine and Biology Society (EMBC), Milan, Italy, 25-29 August 2015; pp. 5179-5182.

14. Acosta-Escalante, F.D.; Beltrán-Naturi, E.; Boll, M.C.; Hernández-Nolasco, J.A.; García, P.P. Meta-Classifiers in Huntington's Disease Patients Classification, Using iPhone's Movement Sensors Placed at the Ankles. IEEE Access 2018, 6, 30942-30957. [CrossRef]

15. Agrawal, S.C.; Tripathi, R.K.; Jalal, A.S. Human-fall detection from an indoor video surveillance. In Proceedings of the 2017 8th International Conference on Computing, Communication and Networking Technologies (ICCCNT), Delhi, India, 3-5 July 2017; pp. 1-5.

16. Ali, A.H.; Razak, M.R.A.; Hidayab, M.; Azman, S.A.; Jasmin, M.Z.M.; Zainol, M.A. Investigation of indoor WIFI radio signal propagation. In Proceedings of the 2010 IEEE Symposium on Industrial Electronics and Applications (ISIEA), Penang, Malaysia, 3-5 October 2010; pp. 117-119.

17. Wang, C.; Chen, S.; Yang, Y.; Hu, F.; Liu, F.; Wu, J. Literature review on wireless sensing-Wi-Fi signal-based recognition of human activities. Tsinghua Sci. Technol. 2018, 23, 203-222. [CrossRef]

18. Adib, F.; Hsu, C.-Y.; Mao, H.; Katabi, D.; Durand, F. Capturing the human figure through a wall. ACM Trans. Graph. 2015, 34, 1-13. [CrossRef]

19. Xi, W.; Huang, D.; Zhao, K.; Yan, Y.; Cai, Y.; Ma, R.; Chen, D. Device-Free Human Activity Recognition Using CSI. In Proceedings of the 1st Workshop ACM, Denver, CO, USA, 16 October 2015.

20. Siriwongpairat, W.P.; Su, W.; Olfat, M.; Liu, K.J.R. Multiband-OFDM MIMO coding framework for UWB communication systems. IEEE Trans. Signal Process. 2006, 54, 214-224. [CrossRef]

21. Khan, M.B.; Yang, X.; Ren, A.; Al-Hababi, M.A.M.; Zhao, N.; Guan, L.; Fan, D.; Shah, S.A. Design of Software Defined Radios Based Platform for Activity Recognition. IEEE Access 2019, 7, 31083-31088. [CrossRef]

22. Edfors, O.; Sandell, M.; van de Beek, J.J.; Wilson, S.K.; Borjesson, P.O. OFDM channel estimation by singular value decomposition. IEEE Trans. Commun. 1998, 46, 931-939. [CrossRef]

23. Leung, C.M.; Chan, Y.W.; Chang, C.M.; Yu, Y.L.; Chen, C.N. Huntington's disease in Chinese: a hypothesis of its origin. J. Neurol. Neurosurg. Psychiatry 1992, 55, 681-684. [CrossRef] [PubMed]

24. Zhang, L.; Qin, Y.; Zhang, J. Study of polynomial curve fitting algorithm for outlier elimination. In Proceedings of the 2011 International Conference on Computer Science and Service System (CSSS), Nanjing, China, 27-29 June 2011; pp. 760-762.

25. Zhang, J.X.; Zhong, Q.-H.; Dai, Y.-P.; Liu, Z. A new de-noising method based on wavelet transform and transforming Hampel filter. In Proceedings of the SICE Conference IEEE, Fukui, Japan, 4-6 August 2003.

26. Yuan, L.; Kesavan, H.K. Minimum entropy and information measure. IEEE Trans. Syst. Man Cybern. Part C Appl. Rev. 1998, 28, 488-491. [CrossRef]

(C) 2020 by the authors. Licensee MDPI, Basel, Switzerland. This article is an open access article distributed under the terms and conditions of the Creative Commons Attribution (CC BY) license (http://creativecommons.org/licenses/by/4.0/). 